\title{
Precise Table Recognition by Making Use of Reference Tables
}

\author{
Claudia Wenzel ${ }^{1}$ and Wolfgang Tersteegen ${ }^{2}$ \\ ${ }^{1}$ German Research Center for Artificial Intelligence (DFKI) \\ P.O. Box 2080, 67608 Kaiserslautern/Germany \\ Claudia.Wenzel@dfki.de \\ ${ }^{2}$ University for Applied Sciences of Emden (FHO) \\ Constantiaplatz 4, 26723 Emden/Germany
}

\begin{abstract}
The ScanTab system represents a knowledge-based approach to table recognition in scanned documents. In contrast to most systems which recognize tables by grouping layout information, our system uses predefined information about which table types may appear in the documents. This enables a very accurate detection able to cope with distorted tables and tables providing little layout information, e.g., no lines, bad alignment, or few rows. Table recognition starts with the detection of the table header. Afterwards, this header is compared with table headers of known reference tables. Having determined the correct reference table, the information kept in the knowledge base is utilized to compute the complete table structure. A graphical user interface allows an easy and fast specification of reference tables.
\end{abstract}

\section{Introduction}

Nowadays, document analysis systems cover all kinds of documents, e.g., business letters, books, technical reports, or bank cheques. In all these documents, special treatment is required for recognizing tables and forms because of their specific structure. Meanwhile, form recognition has become tractable and a lot of approaches capture the fix layout conventions of a form sheet automatically. However, table recognition has different characteristics and is harder to solve. The appearance of a table can vary in different ways, e.g., concerning labels above or beneath a table, nested tables, lines, number of entries.

This paper describes an approach dealing with the analysis of German business letters. In this domain, tables appear quite often to structure offers and orders of goods or services. Some examples can be seen in Figure1. 

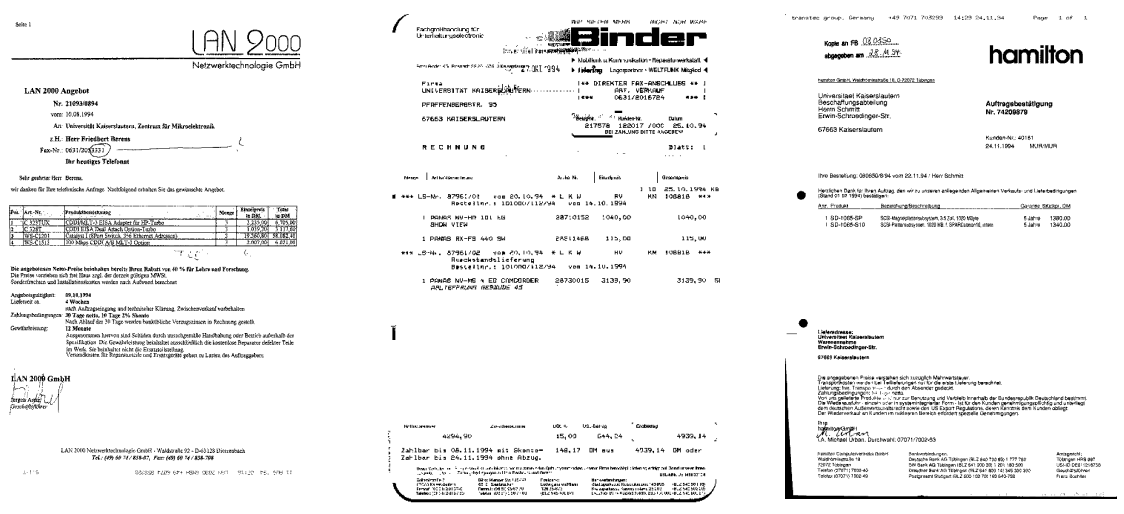

Fig 1. Some exemplary documents containing tables

Our tables are characterized by the following features (revealed by a statistic carried out on 61 documents):

- Most tables have column descriptors (91\%).

- Lines are not mandatory (vertical lines 35\%, horizontal lines for each row $18 \%$, horizontal lines to separate table header and table entries $56 \%$ ).

- Most tables contain up to six columns (68\%) and are small $(37 \%$ with one table entry, only $17 \%$ more than six entries).

- Cell boundaries are violated (41\%) and tables contain text which does not belong to the table $(42 \%)$.

Because of these bad characteristics, we have chosen a knowledge-based approach to table recognition. That means, we require a certain amount of information about the tables in the domain in advance. By this, we are able to reach high precision and recall values despite the bad table quality.

Our ScanTab system is equipped with a graphical user interface which enables work in two modes: During reference table specification, the user specifies table details which are necessary to enter the current table as new table model. During table recognition, the current table is recognized based on the knowledge base of reference tables. Both modes start with the same procedure, namely the detection of the table header. This step requires the existence of labels for table columns. The further analysis respectively the specification of a new reference table is guided by the table header.

Table analysis has become a prominent research topic in document analysis since the early 1990's. Most approaches to table recognition described so far utilize purely geometric features (e.g. pixel distribution, line-art) to determine the logical structure of the table. For example, Hori and Dörmann [1] use a method called "box-driven reasoning" to analyze tables with touching characters and broken lines. It is based on the computation of boxes for e.g., cells, characters, bars, and noise. Relations between boxes are extracted to detect missing cell boxes and touching characters. Chandran 


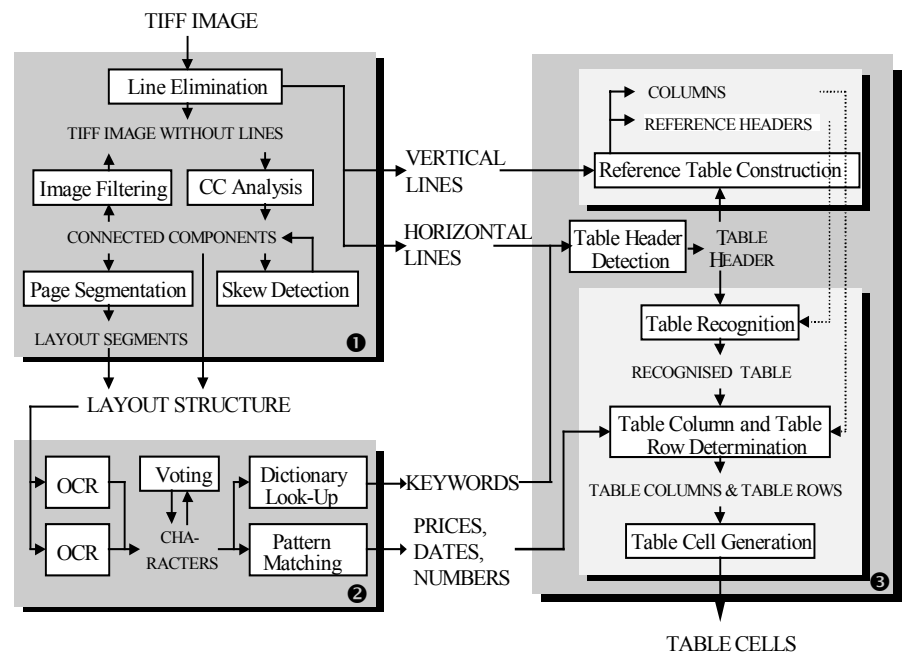

Fig. 2. Processing Model (1) Structure analysis, (2) Text recognition, (3) Table analysis)

and Kasturi [2] use line information to determine a table's location. Afterwards, horizontal and vertical pixel projection reveals white streams as additional cell boundaries. Then, resulting cells are labeled in a specific format as headings, subheadings, or table entries.

Green and Krishnamoorthy [3] employ visual clues (lines, fonts, textual semantics, data format) to divide a table into cells. Using a table model, the tables logical structure is detected in a subsequent step. Consider Hirayama [4] to read more about bottom-up approaches.

The scenario of the work of Shamilian et al [5] is similar to our system. Their "retargetable table reader" recognizes known predefined table styles. A graphical user interface supports the definition of table styles. It consists of selecting the region of the table, determining field boundaries, and assigning several information to the fields such as name, and so on. After this, documents containing tables in a known layout are analyzed by several steps, namely background suppression, elimination of line-art, table location, table record identification, field location, OCR, and contextual analysis. The kernel of the table recognition procedure is based on text-line projection.

\section{Processing Model}

This chapter provides an overview on the table analysis procedure. As shown in Figure 2, all documents (already scanned with 300 dpi) are processed by three steps.

Structure Analysis: First, vertical and horizontal lines are removed to reduce the error rate of the page segmentation. All information about line coordinates and line types is separately stored for further use. Next, a connected component analyzer generates a 
hierarchy of black and white components and uses them to detect background texture or noise in the document. Then, image filtering deletes the texture while all noisy regions are filtered by the morphological opening operation. Afterwards, skew detection determines the dominant skew. Finally, page segmentation classifies the document into text and non text regions and segments regions into block, line, word, and character segments.

Text Recognition: In the text recognition step, the results of two commercial OCR tools are combined by a voting component which provides a higher accuracy. Subsequently, dictionary look-up returns valid word hypotheses based on the weighted edit distance. The dictionary used contains special keywords typically found in table headers. Identification of specific content information (numbers of a fixed format, dates, and prices) is performed by employing finite-state-automata. For more information about structure analysis and text recognition please refer to Baumann et al [6].

Table Analysis: In the third step, table data is extracted. First, the table header detection tries to locate the table header by relating the positions of the keywords to each other. If available, horizontal lines are used to improve the generated result. Secondly, the column information of the table is delivered by comparing the table header of an unknown table with table headers of known tables (so-called reference tables). This information is also used to create rows. Together with the column borders, they result in the cells of the table. Finally, the contents of the corresponding cells are assigned to their logical objects.

A necessary precondition for our recognition algorithm is a knowledge base of documents containing the same table structures as the documents which are analyzed later-on. For entering a document in this knowledge base, its table header must be localized and column information (e.g. positions of column borders) must be entered.

\section{Detecting Table Headers}

The detection of the table header is necessary for table cell generation and reference table construction. It is divided in several steps: After reducing the search area and rotating words, a starting point (called pivot-element) for the table header is determined. This starting point is horizontally expanded to establish table boundaries.

\subsection{Pre-processing}

After the dictionary look-up in the text recognition step is finished, all words are divided into two groups: keywords and other words. Keywords are words contained in the dictionary of known table headers and located within a certain search area. In fact, one can expect different information in special regions of the document, e.g. sender at the top and banking arrangements at the bottom (this is typical for German business letters). Thus, the number of words investigated can be reduced by applying thresholds. Furthermore, pre-processing corrects inclinations by rotating all words within the search area. 


\subsection{Detection Algorithm}

In our domain, table headers consist of a maximum of three text lines. In fact, they often contain only one line. This observation is crucial for the detection algorithm.

The detection starts with the determination of a so-called pivot element. Every keyword is supposed to be the pivot element of a table header, provided it has a minimum number of characters. The pivot element sets the maximum expansion of the table header upwards and downwards (calculated from the left top corner respectively the right bottom corner of the pivot element). All other keywords lying in the area calculated are also members of this potential table header. The table header containing the maximum number of keywords $\left(\max \left[\right.\right.$ header $_{1}, \ldots$, header $\left.\left._{n}\right]\right)$ is the final header. However, only those headers with a certain number of words are taken into consideration.

If at this time the table header is not unique, the algorithm computes sector counters of these headers. A sector counter counts every word (not only keywords) lying in the same line as the pivot element. The table header with the highest sector counter wins the comparison. At the end, the final table header is filled with all remaining words (non keywords) lying in the area of the corresponding pivot element. This compensates OCR mistakes.

The next part aims at localizing horizontal lines which are members of the final table header. There are either no lines, one or two lines (one or none above and one or none below the header). Lines are used to verify the final boundaries of the header. Therefore, the search area of the pivot element is enlarged which is done separately for the area above and below the pivot element. The algorithm looks sequentially at all lines of the document. It finishes as soon as a line of a certain minimum length is lying in the enlarged area.

If the algorithm is not able to find an upper or lower line for setting table boundaries, the appropriate area (above and below the pivot element) is treated by a different procedure. Therefore, all keywords of the area are counted. If the number of keywords is less then a certain threshold value, all words in this area are removed. So the final size of a table header is between one and three lines. The threshold value guarantees that the words in the area analyzed really belong to the table header. The more keywords exist, the more likely this will be.

\subsection{Final Processing}

In case the detection algorithm has been successfully completed, word positions are used by a transformation step to generate rows and columns. The final size of the table header is calculated with help of word boundaries and then adjusted by the upper and lower lines. If the table header's width is too small (related to the width of the document), table header detection has failed. Figure 3 shows an example of a successfully detected table header. 


\begin{tabular}{|c|c|c|c|c|c|c|}
\hline $\begin{array}{l}\text { Posifions } \\
\text { nimimer }\end{array}$ & Meng: & $\begin{array}{l}\text { Marell } \\
\text { numintal }\end{array}$ & Beschreibung & Einzelpreis DMA & $\begin{array}{l}\text { voraussicht } \\
\text { Eieferdatum }\end{array}$ & \\
\hline \multirow[t]{4}{*}{0100} & 1 & A4052A & Upgrades HP9OOD Mdl 720 to Mdl $735 / 125$ & $37.877,00$ & Ende & Nov4 \\
\hline & 1 & 008 & Instalt LAN AUI Interface & 0,00 & & \\
\hline & & & Nachlass $(41.0 \%)$ & $-15.529,57$ & & \\
\hline & & & Postensumme & $22.347,43$ & & \\
\hline \multirow[t]{4}{*}{0200} & 1 & A1986A & 720 Aufrüstkit f. einen EISA-Steckplatz & $3.475,00$ & Ende & Nov4 \\
\hline & 1 & $0 S 4$ & 1nst. und HP LAN-Konfiguration & 609,00 & & \\
\hline & & & Nachlass $(41.0 \%)$ & $-1.674,44$ & & \\
\hline & & & Postensumine & $2.409,56$ & & \\
\hline
\end{tabular}

Fig. 3. Screenshot of a detected table header

\section{Reference Table Construction}

The construction of a reference table starts with the table header detection as already described. It builds the starting point for the following further table specification:

1. OCR errors in the table header can be corrected manually by clicking the incorrect word and typing in the correct one.

2. Vertical table boundaries are generated automatically by using the left boundary of the leftmost word in the table header and by using the right boundary of the rightmost word.

3. Now the user is able to draw vertical lines to correct the table boundaries or to mark boundaries of table columns. Drawing is accomplished by moving the cursor to the intended line position and clicking the right mouse button. Resulting lines are displayed as shown in Figure 3.

4. For each column, the user can specify the orientation of the column entries (left/ right/ none). Moreover, one can specify entries belonging to a certain word class by using regular expressions. This enables the lexical preprocessing to detect and mark such expressions.

5. The user specifies details for the generation of table cells: Each column can be labeled as top-separator, bottom-separator, or repeater. A top-separator is a column in which each line entry marks the beginning of a new table cell (typically for running numbers). A bottom-separator is a column in which each line entry marks the end of a table cell (prices). A repeater column does not contain an entry in each cell. In this case, the intended meaning of the cell is a repetition of the value of the next cell up (dates).

6. Finally, this specification is saved.

Figure 4 shows a screenshot where all details have been specified. The left window reveals the column boundaries and the right window displays more detailed information. Manual effort for the construction of a reference table takes about 3 minutes. 

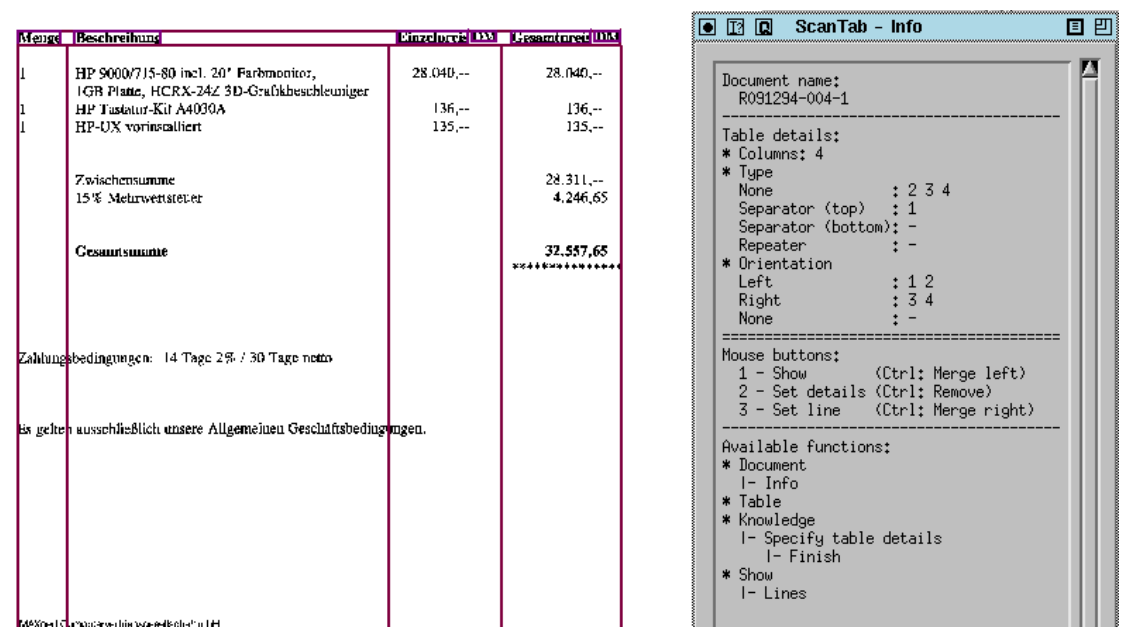

Fig. 4. Final Screenshot for Reference Table Construction

\section{Table Recognition}

Table recognition subsumes the assignment of a reference table to an unknown table. It is based on the following observation: It is impossible that two table headers belonging to different table types are identical with regard to keywords and the distances between these keywords. Thus, table recognition is based on a comparison of the table header of the unknown table with table headers of reference tables. In case of successful comparison, information about the table structure can be retrieved from the knowledge base of reference tables and is used for further analysis.

The following subsections describe the corresponding algorithms in more detail.

\subsection{Recognition Algorithm}

The table header of an unknown table is sequentially compared with all available table headers of the knowledge base. For a final assignment of a reference table, the hereby determined best similarity must be higher than a certain threshold value.

The comparison of two table headers consists of five phases, whereas phase one and phase two only provide yes/no answers which decide on the execution of phase three, four, and five. As a result of that the search area is considerably reduced to speed up the overall procedure. The final result of the recognition procedure is an assignment to a reference table. 
(1) Comparison of the number of words: If the number of all words contained in the table headers differs too strongly from each other, it cannot be expected that the table headers match each other. However, a certain tolerance is necessary because the number of words in the same table header is subject to minor changes due to segmentation errors or slight changes in the layout style.

(2) Comparison of the keywords: First, the keywords in the table headers are sorted by the quicksort algorithm to improve efficiency. Then, all keywords being part of the first $\left(\mathrm{H}_{1}\right)$ as well as the second header $\left(\mathrm{H}_{2}\right)$ are inserted in the temporary headers $\mathrm{TH}_{1}$ and $\mathrm{TH}_{2}$. So at the end $\mathrm{H}_{1}$ and $\mathrm{H}_{2}$ contain only words which have no equivalent word in the other table header. If one header contains several equivalent words for one word of the other header, the word is chosen which has the least distance to the partner word of the other table header. This phase fails either if the number of the remaining words (that means words without an equivalent) exceeds a certain value or if too few pairs exist.

In order to determine the shift between both headers all word pairs detected are investigated. By calculating an average value out of the distances between the word pairs the influence of some deviating values is decreased. At the end of this phase, $\mathrm{TH}_{1}$ and $\mathrm{TH}_{2}$ contain only keywords appearing in both headers.

(3) Distance check: For each word in the headers $\mathrm{TH}_{1}$ and $\mathrm{TH}_{2}$ the distance to all other words of the same header is determined. This produces a kind of distance network for the keywords. Next, a counter registers all distances in $\mathrm{TH}_{1}$ which differ too strongly from the corresponding distances in $\mathrm{TH}_{2}$. The degree of similarity results from the relation between the final counter value and the overall number of comparisons (used for standardization).

(4) Pattern matching: Goal of this phase is to check the remaining words in $\mathrm{H}_{1}$ and $\mathrm{H}_{2}$ (remember phase two) which could not be assigned to a word pair. The following procedure is executed for each word in $\mathrm{H}_{1}$ with all words in $\mathrm{H}_{2}$. The check of a pair is done by comparing

1. the similarity of the horizontal and vertical size proportions of the word segments

2. the distances of the word out of $\mathrm{H}_{1}$ to all keywords in $\mathrm{TH}_{1}$ with the distances of the word out of $\mathrm{H}_{2}$ to all other words in $\mathrm{TH}_{2}$.

The average value of the size and distance correspondence delivers the similarity of both words. If the best similarity exceeds a certain threshold, the comparison was successful and the words are removed from $\mathrm{H}_{1}$ and $\mathrm{H}_{2}$.

In case $\mathrm{H}_{1}$ and $\mathrm{H}_{2}$ are finally empty, the degree of similarity is set to one. Otherwise, the number of unreferenced words is determined for every table column and weighted according to its share in the total quantity of contained words. The sum of all weights related to the number of words (before execution of phase four) in the bigger one of the two headers $\mathrm{H}_{1}$ and $\mathrm{H}_{2}$ supplies the similarity degree.

(5) Calculation of the total similarity: In case all previous phases have been finished, the total similarity of the two headers is calculated on the basis of the similarity values gained in phase three and four. This is done by weighting them differently: 


$$
\text { similarity }_{\text {total }}^{\circ}=\frac{\left[\sum \text { Words }\left(\mathrm{TH}_{1}\right) * \text { similarity }_{\text {phase } 3}^{\circ}+\max \left(\sum \operatorname{Words}\left(\mathrm{H}_{1}\right), \sum \operatorname{Words}_{2}\left(\mathrm{H}_{2}\right)\right) * \text { similarity }_{\text {phase }}^{\circ}\right.}{\left[\sum \operatorname{Words}\left(\mathrm{TH}_{1}\right)+\max \left(\sum \operatorname{Words}\left(\mathrm{H}_{1}\right), \sum \operatorname{Words}\left(\mathrm{H}_{2}\right)\right)\right]}
$$

Note that in the formula the number of words of $\mathrm{H}_{1}$ and $\mathrm{H}_{2}$ is calculated before execution of phase four.

\subsection{Generating Table Cells}

Reference table information can be used in the following way for cell generation:

- Shift between both table headers

- Coordinates of column rows (relative to the table header)

- Details about special columns containing separators and repeaters.

With this information, table cells are generated by using intersections between column lines and row lines caused by the separators. By exclusive treatment of columns with special patterns and by considering the column alignment, influence of text which does not belong to the table can be reduced.

Determination of special columns: In phase one, the special patterns are assigned to those columns with a particular type (separator and repeater). All remaining special patterns are treated as normal words. Since it must be expected that some of the coordinates of the column positions are slightly inaccurate, patterns are also accepted if they start or end outside the column borders (only within a certain tolerance).

Filtering of the special columns: Now all special columns are filtered:

1. For each pattern in a column it is counted how many other patterns of this column start (left aligned) respectively end (right aligned) at the same position as the pattern itself (including a certain tolerance once more). The pattern with the highest counter sets the so-called alignment point.

2. All pattern of the column with a different alignment point (again in the tolerance range) are removed.

3. Normal words with the same alignment point - lying in the tolerance range - which fit into the column are included in the column.

Creation of row lines: Row line creation is done on the basis of the special columns. In order to determine whether a row line starts or ends a table entry, special columns of the type separator are divided in two classes: The first class contains columns where data are at the beginning of an entry while the second class contains data at the end of an entry. A filter unites all lines with a distance less than a certain number of pixels. It is important that both sorts of row lines are filtered separately, because the distance between lines which start an entry and lines which end an entry is normally very small. Of course, if there is only one column with starting lines (resp. ending lines), the filtering is omitted. 
Assignment of cell contents: Intersections between column lines and row lines produce the table cells. Afterwards, table data is assigned to them by first processing all cells with data out of special columns. Then all normal words are used to fill the remaining cells.

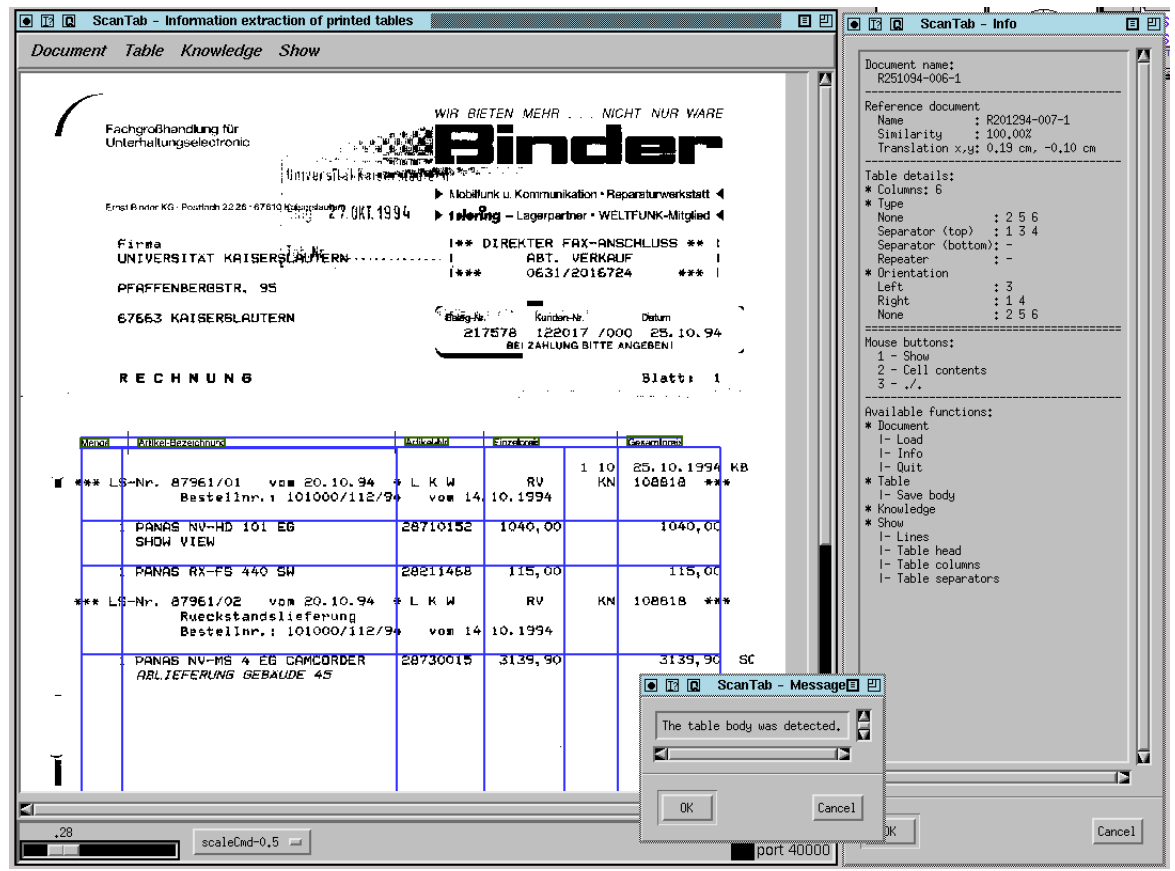

Fig. 5. Screenshot of a table completely analyzed (partial view)

Note that there is also a tolerance range for the dimensions of the table cells. To conclude, Figure 5 shows the result after a complete table analysis.

\section{Results}

Up to now, our system has been used on a comparatively small set of 99 documents collected at the purchasing department of the University of Kaiserslautern. However, the system's processing steps were determined by testing it on a much larger set but we did not measure the behavior in figures. The set of 99 business letters was divided into three different document clusters:

- 24 documents were used to build up the knowledge base with reference tables (training set)

- 44 documents served as test set for the full analysis process (test set)

- 31 documents were the basis for a special test of the table header detection (without the table recognition) under aggravated circumstances, i.e., we have chosen 
documents with a high error rate regarding to segmentation and/or character recognition (special set)

Table 1 presents the evaluation results of tests with the test set and the special set in terms of recall and precision.

Table 1. Test results

\begin{tabular}{|c|c|c|}
\hline Document Cluster & Precision & Recall \\
\hline test set & $100 \%$ & $96 \%$ \\
\hline special set & $100 \%$ & $100 \%$ \\
\hline
\end{tabular}

During the test of the whole analysis procedure for every document out of the training set, two (if available, otherwise one) belonging documents out of the test set with the same table header were processed. Precondition for all documents was that the correctness of the keyword segmentation had to be better than $75 \%$.

The technique for the generation of the table cells delivered good results if no text which does not belong to the table was present. However, if this requirement was not met then the results were often inaccurate. Since due to this distinction the results of cell recognition cannot be classified as correct or incorrect, the precision and recall values are only valid for table header detection and table recognition. So the values for the entire test would be worse if the results for the generation of the table cells would be taken into consideration.

\section{Conclusion}

We have proposed a system for extracting information of tabular structures. It is especially tailored for tables with little layout conventions and tables containing text not belonging to the table and violating the table layout. To reach a high accuracy, our system is based on the assignment of predefined reference tables to new unknown tables. Moreover, the definition of reference tables allows to integrate information extraction from tabular structures into an extraction procedure for the whole document by assigning semantic labels to columns.

In our opinion, our approach seems very promising. Table header determination and reference table assignment can serve as a very accurate starting point for further analysis. The system tests delivered very good results. However, due to the small basis of documents they cannot be seen as representative. For that purpose it would be necessary to use considerably more documents in another test. The table cell generation needs yet some improvements in order to improve the ability to handle tables with text which does not belong to the table. Furthermore, this procedure can be refined by incorporating ruled line information and a more accurate determination of the table end. 


\section{Acknowledgements}

This work has been supported by the German federal ministry of education, science, research and technology under contract number 01 IW 807.

\section{References}

1. O. Hori, D. S. Dörmann: Robust Table-form Structure Analysis Based on BoxDriven Reasoning. Proceedings of the International Conference on Document Analysis and Recognition (ICDAR 95)

2. S. Chandran, R. Kasturi: Structural Recognition of Tabulated Data. Proceedings of the International Conference on Document Analysis and Recognition (ICDAR 93)

3. E. Green, M. Krishnamoorthy: Model-Based Analysis of Printed Tables. Proceedings of the International Conference on Document Analysis and Recognition (ICDAR 95)

4. Y. Hirayama: A Method For Table Structure Analysis Using DP Matching. Proceedings of the International Conference on Document Analysis and Recognition (ICDAR 95)

5. J. Shamilian, H. Baird, T. Wood: A Retargetable Table Reader. Proceedings of the International Conference on Document Analysis and Recognition (ICDAR 97)

6. S. Baumann, M. Ben Hadj Ali, A. Dengel, T. Jäger, M. Malburg, A. Weigel, C. Wenzel: Message Extraction from Printed Documents - A Complete Solution. Proceedings of the International Conference on Document Analysis and Recognition (ICDAR 97) 